\title{
SIGN CHANGES OF HECKE EIGENVALUES
}

\author{
KAISA MATOMÄKI AND MAKSYM RADZIWIŁŁ
}

\section{INTRODUCTION}

Let $f$ be a holomorphic Hecke cusp form or a Maass Hecke cusp form and write $\lambda_{f}(n)$ for its normalized Fourier coefficients, so that

$$
f(z)=\sum_{n=1}^{\infty} \lambda_{f}(n) n^{(\kappa-1) / 2} e(n z)
$$

in the holomorphic case, and

$$
f(z)=\sqrt{y} \sum_{n \neq 0} \lambda_{f}(n) K_{s-1 / 2}(2 \pi|n| y) e(n x)
$$

in the Maass case.

In [14] Kowalski, Lau, Soundararajan and Wu investigate the problem of the first sign change of $\lambda_{f}(n)$ for holomorphic $f$. They remark on the similarities with the problem of the least quadratic residue. This motivates the point of view that the signs of $\lambda_{f}(n)$ are GL(2) analogues of real characters. The frequency of signs and sign changes and other related questions have been also recently studied in 1, 15, 16, 18, 23]. In [5] Ghosh and Sarnak relate sign changes of $\lambda_{f}(n)$ to zeros of $f(z)$ on the imaginary line.

The sequence of signs of $\lambda_{f}(n)$ alone is known to determine the form $f$ (see [14]). Because of this we expect a significant amount of randomness in the distribution of the signs of $\lambda_{f}(n)$. It is the finer details of this randomness that we set out to investigate in this paper. We start by showing that half of the non-zero $\lambda_{f}(n)$ are positive and half are negative. This is relatively straightforward and depends only on the multiplicativity of $\lambda_{f}(n)$. For simplicity we focus on the case of the full modular group.

Theorem 1.1. Let $f$ be a holomorphic Hecke cusp form for the full modular group Asymptotically half of the non-zero $\lambda_{f}(n)$ are positive and half of them are negative.

Date: April 23, 2015.

The first author was supported by the Academy of Finland grant no. 137883. The second author was partially supported by NSF grant DMS-1128155. 
Theorem 1.1 was also recently obtained independently (for Maass forms) by Elliott and Kish [2, Theorem 7]. Previously it was known (for holomorphic forms) that a positive proportion of the $\lambda_{f}(n)$ are positive and a positive proportion negative [16. Theorem 1]. If $\lambda_{f}(n)$ and $\lambda_{f}(n+1)$ behave independently, and $\lambda_{f}(n)$ never vanishes then we expect $(1 / 2+o(1)) x$ sign changes in the sequence $\left(\lambda_{f}(n)\right)_{n \leq x}$, since individually each $\lambda_{f}(n)$ and $\lambda_{f}(n+1)$ is positive and negative half of the time. Even if $\lambda_{f}(n)$ happened to vanish we still expect a positive proportion of sign changes on the relative subset of those $n$ for which $\lambda_{f}(n) \neq 0$. Note that we do not consider the situation where $\lambda_{f}(n)<0$ and $\lambda_{f}(n+1)=0$ to be a sign change, because the sign of 0 is undefined. Thus, by number of sign changes of a possibly vanishing sequence $\lambda_{f}(n)$ we mean the number of sign changes of $\lambda_{f}(n)$ on the subset of $n$ at which $\lambda_{f}(n) \neq 0$. Our main result is the following.

Theorem 1.2. Let $f$ be a holomorphic Hecke cusp form for the full modular group or a Hecke Maass cusp form for the full modular group. Then for every large enough $x$, the number of sign changes in the sequence $\left(\lambda_{f}(n)\right)_{n \leq x}$ is of the order of magnitude

$$
\#\left\{n \leq x: \lambda_{f}(n) \neq 0\right\} \asymp x \prod_{\substack{p \leq x \\ \lambda_{f}(p)=0}}\left(1-\frac{1}{p}\right) .
$$

For holomorphic forms $f$ Serre [21] established that $\lambda_{f}(n) \neq 0$ for a positive proportion of $n$. This is expected to hold for Hecke Maass forms of eigenvalue $>\frac{1}{4}$ but is not currently known. By comparing the second and fourth moment of $\left|\lambda_{f}(n)\right|$ with $f$ a Hecke Maass form, one can show that $\lambda_{f}(n) \neq 0$ for at least $c X / \log X$ integers $n \leq X$. Slightly better results can be obtained by comparing moments of $\left|\lambda_{f}(p)\right|$.

Previously it was only known that there are $\gg x^{1 / 2}$ sign changes in the holomorphic case (see [16]) and $x^{1 / 8-\varepsilon}$ sign changes in the Maass case (see [1]). We will prove in Section 7 the following corollary on a variant of Chowla's conjecture.

Corollary 1.3. Let $f$ be a holomorphic Hecke cusp form for the full modular group. There exists a constant $c>0$ such that, for all $x$ large enough,

$$
\left|\sum_{n \leq x} \operatorname{sgn}\left(\lambda_{f}(n)\right) \operatorname{sgn}\left(\lambda_{f}(n+1)\right)\right| \leq(1-c) x .
$$

This is non-trivial when all of the $\lambda_{f}(n)$ are non-zero. It would be interesting to rule out the possibility of $\lambda_{f}(n)$ regularly flipping its sign and thus to investigate the entropy of the sequence of sign of $\lambda_{f}(n)$.

According to the Sato-Tate conjecture (now a theorem), for any $I \subset[0,2]$ there is a positive proportion of primes $p$ such that $\left|\lambda_{f}(p)\right| \in I$. Because of this the size of $\left|\lambda_{f}(n)\right|$ fluctuates wildly, making it hard to detect sign changes. This is similar to 
a problem one encounters when studying the zeros of the Riemann zeta-function on the half-line. In analogy to that problem, we introduce sieve weights $w_{n} \geq w_{n}^{\prime} \geq 0$ and compare

$$
\left|\sum_{x \leq n \leq x+h} \operatorname{sgn}\left(\lambda_{f}(n)\right) w_{n}\right| \text { and } \sum_{x \leq n \leq x+h} w_{n}^{\prime}
$$

with the intent of showing that the first term is frequently less than the second (since this ensures a sign change in the interval $[x, x+h]$ ). To obtain cancellations in the first sum in (2) (for almost all $x$ ), we use the work of Harcos on the shifted convolution problem [8],

$$
\sum_{\substack{X \leq m, n \leq 2 X \\ a m-b n=h}} \lambda_{f}(n) \lambda_{f}(m)
$$

(see also [20, Section 3] for the best current result). The choice of sieve weights $w_{n}$ allows us to introduce a bilinear structure, and to bound (2) on average by picking out the cancellations in (3) coming from the large primes, while ignoring the small primes (which are a source of problem due to the size fluctuation of $\left|\lambda_{f}(n)\right|$ when $n$ has many small prime factors). To obtain a good lower bound for the second, non-oscillating, sum in (2) for a positive proportion of $x$ we compare the first and second moments,

$$
\sum_{X \leq n \leq 2 X} w_{n}^{\prime} \text { and } \sum_{X \leq n \leq 2 X} w_{n}^{\prime 2}
$$

The computation of these moments relies on the close resemblence of $w_{n}^{\prime}$ to a multiplicative function supported on the large primes.

Finally Theorem [1.2 does not need to be limited to the full modular group as long as coefficients of the form are real. For holomorphic forms $f$ with complex multiplication, Serre has shown that [21]

$$
\sum_{\substack{p \leq x \\ \lambda_{f}(p)=0}} \frac{1}{p}=\frac{1}{2} \log \log x+O(1) .
$$

A variant of Theorem 1.2 could be used to show that, conditioned on the set of those $n \leq x$ for which $\lambda_{f}(n) \neq 0$, there is a positive proportion of sign changes. We end our discussion by posing the following problem.

Problem. Let $f$ be a Hecke Maass cusp form of eigenvalue $>\frac{1}{4}$. Is it possible to show that the coefficients $\lambda_{f}(n)$ are not lacunary? Precisely is it possible to show that

$$
\sum_{\lambda_{f}(p)=0} \frac{1}{p}<\infty ?
$$


We remark that one could work out explicitly the proportion of sign changes that Theorem 1.2 yields, although we expect that doing so is rather hard. It might also be possible to apply our techniques to study sign changes of the error term in the number of representation of $n$ by a quadratic form in $2 k$ variables. However to obtain, when applicable, a good result (such as a positive proportion of sign changes) would require adapting some of the techniques of Selberg designed to study zeros of linear combinations of $L$-functions, which would increase the complexity of the proof.

The plan of the paper is as follows: In Section 2 we prove Theorem 1.1 using properties of general multiplicative functions. In Section 3 we formulate three propositions and show how our main result, Theorem 1.2 follows from them. These three propositions are then proved in Sections $4,6$.

Remark 1.4. While this paper was being refereed, we have been able to obtain (by completely different means) a rather general result on multiplicative function in short intervals [17] which implies among other things that a multiplicative function $f: \mathbb{N} \rightarrow \mathbb{R}$ has a positive proportion of sign changes as soon as $f(m)<0$ for some integer $m$ and $f(n) \neq 0$ for a positive proportion of integers $n$. This recovers Theorem 1.2 in the holomorphic case, but not in the case of Maass forms, since for a Maass form it is currently not ruled out that $\lambda_{f}(n)=0$ for almost all integers $n$. In addition as pointed out by the anonymous referee, the method developed in this paper is general and will work for any multiplicative function satisfying reasonable estimates for the associated shifted convolution problem, which is especially interesting when the function vanishes on many primes.

\section{Proof of Theorem 1.1}

Theorem 1.1 follows quickly from the following lemma.

Lemma 2.1. Let $K, L: \mathbb{R}_{+} \rightarrow \mathbb{R}_{+}$be such that $K(x) \rightarrow 0$ and $L(x) \rightarrow \infty$ for $x \rightarrow \infty$. Let $g: \mathbb{N} \rightarrow\{-1,0,1\}$ be a multiplicative function such that, for every $x \geq 2$,

$$
\sum_{\substack{p \geq x \\ g(p)=0}} \frac{1}{p} \leq K(x) \quad \text { and } \quad \sum_{\substack{p \leq x \\ g(p)=-1}} \frac{1}{p} \geq L(x) .
$$

Then

$$
\begin{aligned}
|\{n \leq x: g(n)=1\}| & =(1+o(1))|\{n \leq x: g(n)=-1\}| \\
& =\left(\frac{1}{2}+o(1)\right) x \prod_{p \in \mathbb{P}}\left(1-\frac{1}{p}\right)\left(1+\frac{|g(p)|}{p}+\frac{\left|g\left(p^{2}\right)\right|}{p^{2}}+\cdots\right),
\end{aligned}
$$


where $o(1) \rightarrow 0$ when $x \rightarrow \infty$ and the rate of convergence depends only on $K(x)$ and $L(x)$ but not on $g$.

For the proof of this lemma, we use two results concerning averages of multiplicative functions. The first lemma (see [7] though a good enough result for our purposes would also quickly follow from Halász's theorem) shows that a real-valued multiplicative function $g: \mathbb{N} \rightarrow[-1,1]$ is small on average unless it "pretends" to be the constant function 1 .

Lemma 2.2. There exists an absolute positive constant $C$ such that, for any multiplicative function $g$ with $-1 \leq g(n) \leq 1$, one has

$$
\sum_{n \leq x} g(n) \leq C \cdot x \exp \left(-\frac{1}{4} \sum_{p \leq x} \frac{1-g(p)}{p}\right)
$$

for all $x \geq 2$.

The second lemma concerns average of a positive-valued multiplicative function.

Lemma 2.3. Let $\varepsilon>0$ and let $K: \mathbb{R}_{+} \rightarrow \mathbb{R}_{+}$be such that $K(x) \rightarrow 0$ for $x \rightarrow \infty$. There exists a positive constant $x_{0}$ (depending on $\varepsilon$ and $K(x)$ ) such that if $g: \mathbb{N} \rightarrow$ $[0,1]$ is any multiplicative function for which $\sum_{p>x} \frac{1-g(p)}{p} \leq K(x)$ for all $x \geq 1$, then

$$
\left|\sum_{n \leq x} g(n)-x M(g)\right|<\varepsilon x \quad \text { for all } x \geq x_{0},
$$

where

$$
M(g)=\prod_{p \in \mathbb{P}}\left(1-\frac{1}{p}\right)\left(1+\frac{g(p)}{p}+\frac{g\left(p^{2}\right)}{p^{2}}+\cdots\right) .
$$

Proof. A version which is non-uniform in $g$ can be found in [22, Theorem 11 in Section I.3.8]. However it is easy to see from the proof that the claimed uniformity holds.

Proof of Lemma 2.1. Lemma 2.2 implies that $\sum_{n \leq x} g(n)=o(x)$ and Lemma 2.3 that

$$
\sum_{n \leq x}|g(n)|=(1+o(1)) x \prod_{p \in \mathbb{P}}\left(1-\frac{1}{p}\right)\left(1+\frac{|g(p)|}{p}+\frac{\left|g\left(p^{2}\right)\right|}{p^{2}}+\cdots\right)
$$

so the claim follows since $g(n)$ only takes values in $\{-1,0,1\}$.

Lemma 2.1 immediately implies the following result on signs of multiplicative functions. 
Lemma 2.4. Let $K, L: \mathbb{R}_{+} \rightarrow \mathbb{R}_{+}$be such that $K(x) \rightarrow 0$ and $L(x) \rightarrow \infty$ for $x \rightarrow \infty$. Let $g: \mathbb{N} \rightarrow \mathbb{R}$ be a multiplicative function such that, for every $x \geq 2$,

$$
\sum_{\substack{p \geq x \\ g(p)=0}} \frac{1}{p} \leq K(x) \quad \text { and } \quad \sum_{\substack{p \leq x \\ g(p)<0}} \frac{1}{p} \geq L(x) .
$$

Then

$$
\begin{aligned}
|\{n \leq x: g(n)>0\}| & =(1+o(1))|\{n \leq x: g(n)<0\}| \\
& =\left(\frac{1}{2}+o(1)\right) x \prod_{p \in \mathbb{P}}\left(1-\frac{1}{p}\right)\left(1+\frac{h(p)}{p}+\frac{h\left(p^{2}\right)}{p^{2}}+\cdots\right),
\end{aligned}
$$

where $h(n)$ is the characteristic function of those $n$ for which $g(n) \neq 0$, and o $(1) \rightarrow 0$ when $x \rightarrow \infty$ and the rate of convergence depends only on $K(x)$ and $L(x)$ but not on $g$.

Proof. Apply Lemma 2.1 to the multiplicative function which is 0 for those $n$ for which $g(n)=0$ and $g(n) /|g(n)|$ for those $n$ for which $g(n) \neq 0$.

To prove the long interval result we still need the following lemma.

Lemma 2.5. One has $\lambda_{f}(p)<0$ for a positive proportion of primes.

Proof. This is a direct consequence of the Sato-Tate Conjecture, but follows also without such deep information for instance from [19, Theorem 4(iii)] (even with $m=0$ there).

Proof of Theorem 1.1. Theorem 1.1 follows immediately from Lemma 2.4 together with non-lacunarity and Lemma 2.5.

\section{Outline of the PRoof of Theorem 1.2 (SIgn Changes)}

Let us start by collecting some basic facts about $\lambda_{f}(n)$ which will recur through the argument.

Lemma 3.1. Let $f$ be a holomorphic Hecke cusp form for the full modular group or a Maass Hecke cusp form for the full modular group. Write $\lambda_{f}(n)$ for the normalized Fourier coefficients. Then

(i)

$$
\#\left\{n \leq x: \lambda_{f}(n) \neq 0\right\} \asymp x \prod_{\substack{p \leq x \\ \lambda_{f}(p)=0}}\left(1-\frac{1}{p}\right) \asymp x \prod_{\substack{p \leq x \\ \lambda_{f}(p)=0}}\left(1+\frac{1}{p}\right)^{-1} .
$$

(ii) $\lambda_{f}(n) \ll n^{7 / 64}$. 
(iii) For every $z \geq w \geq 2$,

$$
\sum_{w \leq p \leq z} \frac{\left|\lambda_{f}(p)\right|^{2}}{p}=\sum_{w \leq p \leq z} \frac{1}{p}+O_{f}(1)
$$

(iv) For every large enough $y$,

$$
\sum_{\substack{y \leq p \leq 2 y \\\left|\lambda_{f}(p)\right| \geq 1 / 2}}\left|\lambda_{f}(p)\right| \geq \frac{y}{10 \log y} .
$$

Proof. (i) The second asymptotic equality is trivial, so it is enough to prove the first one. The upper bound is an immediate consequence of an upper bound sieve and the lower bound follows for instance from [6, Theorem 1] together with Lemma 2.5 .

(ii) See [12, Appendix 2].

(iii) By Hecke relation, and since $\lambda_{f}(p)$ are real,

$$
\sum_{w \leq p \leq z} \frac{\left|\lambda_{f}(p)\right|^{2}}{p}=\sum_{w \leq p \leq z} \frac{\lambda_{f}\left(p^{2}\right)+1}{p}
$$

and the claim follows since the second symmetric power $L$-function is cuspidal automorphic (by [4) and thus holomorphic and non-vanishing at $s=1$ (see e.g. [11, Section 5.12]).

(iv) The proof is very similar to [14, Lemma 4.1]. Let us first note that

$$
\frac{1}{8}\left(1+\left(x^{2}-1\right)-\left(x^{4}-3 x^{2}+1\right)\right)=-\frac{x^{4}}{8}+\frac{x^{2}}{2}-\frac{1}{8} \leq \begin{cases}0 & \text { if }|x| \leq 1 / 2 \\ \frac{1}{2} & \text { if }|x|>1 / 2\end{cases}
$$

so that

$$
\begin{aligned}
& \sum_{\substack{y \leq p \leq 2 y \\
\lambda_{f}(p) \mid \geq 1 / 2}}\left|\lambda_{f}(p)\right| \geq \frac{1}{8} \sum_{y \leq p \leq 2 y} 1+\left(\lambda_{f}(p)^{2}-1\right)-\left(\lambda_{f}(p)^{4}-3 \lambda_{f}(p)^{2}+1\right) \\
= & \frac{1}{8} \sum_{y \leq p \leq 2 y} 1+\lambda_{f}\left(p^{2}\right)-\lambda_{f}\left(p^{4}\right)=\left(\frac{1}{8}+o(1)\right) \frac{y}{\log y},
\end{aligned}
$$

by holomorphy and non-vanishing of second and fourth symmetric power $L$ functions (see [12] and [13, Page 194]).

In this section we prove Theorem 1.2 assuming propositions which we will prove in Sections 46. As described in the introduction, the basic idea is to show incompatible bounds for mollified short interval sums (2). Let us start by fixing our choices of $w_{n}$ and $w_{n}^{\prime}$ and the associated notation. 
Fix $X \geq 1$ and set $y=X^{\delta}$ for some small enough $\delta$. Moreover set

$$
\mathcal{D}^{+}=\left\{d=p_{1} \cdots p_{r}: p_{r}<\cdots<p_{1}, p_{m} \leq y_{m} \text { for all odd } m\right\},
$$

where $y_{m}=y^{\frac{1}{2}\left(1-\gamma^{2}\right) \gamma^{m-1}}$ with a parameter $\gamma \in(0,1)$, so that $\mathcal{D}^{+} \subset[1, y]$. Now $\rho^{+}(n)=\sum_{d \mid n, d \in \mathcal{D}^{+}} \mu(d)$ are Brun's sieve weights, so that writing $P^{-}(n)$ for the smallest prime factor of $n$, we have

$$
\mathbf{1}_{P^{-}(n)>y} \leq \rho^{+}(n) .
$$

In particular $\rho^{+}(n) \geq 0$ for all $n$. We then define

$$
w_{n}:=\sum_{\substack{a b=n \\ a \leq y, \lambda_{f}(a) \neq 0 \\(a, b)=1}} \mu^{2}(a) \rho^{+}(b)\left|\lambda_{f}(b)\right|
$$

and

$$
w_{n}^{\prime}:=\sum_{\substack{a b=n \\ a \leq y, \lambda_{f}(a) \neq 0 \\ P^{-}(b)>y}} \mu^{2}(a)\left|\lambda_{f}(b)\right|
$$

Write also

$$
k(x)=\prod_{\substack{p \leq x \\ \lambda_{f}(p)=0}}\left(1+\frac{1}{p}\right), \quad \text { so that } \sum_{\substack{n \leq x \\ \lambda_{f}(n) \neq 0}} 1 \asymp X / k(x)
$$

by Lemma 3.1(i).

Our goal is to compare the sums

$$
\sum_{x \leq n \leq x+h k(x)} \operatorname{sgn}\left(\lambda_{f}(n)\right) w_{n} \text { and } \sum_{\substack{x \leq n \leq x+h k(x) \\ \lambda_{f}(n) \neq 0}} w_{n}^{\prime}
$$

for a large constant $h$. Note that $w_{n}^{\prime} \leq w_{n}$. Therefore if the first sum is smaller in absolute value then we have detected a sign change. Note also that $w_{n}^{\prime}$ consists of only one term. We first need two results on moments of $w_{n}^{\prime}$ and $w_{n}$. From these we will then deduce the behavior of the sums in (4) and the main result will then follow shortly. We will prove the propositions below in sections 4 and 5 respectively.

Proposition 3.2. We have, for all $x>0$ large enough,

$$
\sum_{X \leq n \leq 2 X} w_{n}^{\prime} \gg X \prod_{\substack{p \leq X \\ \lambda_{f}(p)=0}}\left(1-\frac{1}{p}\right) \quad \text { and } \sum_{\substack{X \leq n \leq 2 X \\ \lambda_{f}(p)=0}} w_{n}^{2} \ll X \prod_{\substack{p \leq X \\ \lambda_{f}\left(1-\frac{1}{p}\right)}}(1
$$


Note that the second moment bound is trivial when $f$ is holomorphic since if $P^{-}(b)>y=X^{\delta}$ then $\left|\lambda_{f}(b)\right| \leq \tau(b) \ll 2^{1 / \delta}$ and consequently $w_{n}^{\prime} \ll 2^{2 / \delta}$. Also for holomorphic forms $\sum_{\lambda_{f}(p)=0} 1 / p<\infty$ by a result of Serre. However the second moment estimate is less trivial for Maass forms. We also establish a bound for the second moment of $w_{n}$ which constitutes the technically hardest part of our proof.

Proposition 3.3. We have,

$$
\sum_{X \leq n \leq 2 X} w_{n}^{2} \ll X \prod_{\substack{p \leq X \\ \lambda_{f}(p)=0}}\left(1-\frac{1}{p}\right) .
$$

From Proposition 3.3 and an estimate for the shifted convolution problem of $\lambda_{f}(n)$ we deduce the following proposition.

Proposition 3.4. There exist positive constants $C$ and $\varepsilon$ such that, uniformly in $K>0$ and $h \leq X^{\varepsilon}$, one has, for at least proportion $\left(1-1 / K^{2}\right)$ of $x \in[X, 2 X]$,

$$
\left|\sum_{x \leq n \leq x+h k(x)} \operatorname{sgn}\left(\lambda_{f}(n)\right) w_{n}\right| \leq C K \sqrt{h} .
$$

On the other hand from Proposition 3.2 we deduce the following complimentary result.

Proposition 3.5. For any $h \geq 1$, there is a positive proportion of $x \in[X, 2 X]$ such that

$$
\sum_{x \leq n \leq x+h k(X)} w_{n}^{\prime} \gg h
$$

Proof. Let $\varepsilon$ be a small positive constant and let $\mathcal{H}_{1}$ be the set of square-free integers $n \in[X, 2 X]$ for which $w_{n}^{\prime} \geq \varepsilon$. Then we have by the first part of Proposition 3.2 ,

$$
\sum_{n \in \mathcal{H}_{1}} w_{n}^{\prime} \geq \sum_{X \leq n \leq 2 X} w_{n}^{\prime}-\varepsilon \sum_{\substack{X \leq n \leq 2 X \\ w_{n}^{\prime} \neq 0}} 1 \gg X \prod_{\substack{p \leq X \\ \lambda_{f}(p)=0}}\left(1-\frac{1}{p}\right)
$$

Hence by Cauchy-Schwarz and the second part of Proposition 3.2 ,

$$
\left|\mathcal{H}_{1}\right| \geq \frac{\left(\sum_{n \in \mathcal{H}_{1}} w_{n}^{\prime}\right)^{2}}{\sum_{n} w_{n}^{\prime 2}} \geq \frac{\delta X}{k(x)}
$$


for some $\delta>0$. Consider now the following sets

$$
\begin{aligned}
& \mathcal{U}_{0}:=\left\{X \leq x \leq 2 X:\left|[x, x+h k(X)] \cap \mathcal{H}_{1}\right| \leq \delta h / 2\right\} \\
& \mathcal{U}_{1}:=\left\{X \leq x \leq 2 X: \frac{h}{\delta^{2}} \geq\left|[x, x+h k(X)] \cap \mathcal{H}_{1}\right|>\delta h / 2\right\} \\
& \mathcal{U}_{2}:=\left\{X \leq x \leq 2 X:\left|[x, x+h k(X)] \cap \mathcal{H}_{1}\right| \geq \frac{h}{\delta^{2}}\right\}
\end{aligned}
$$

Then by (6)

$$
\begin{aligned}
\delta h X & \leq \sum_{X \leq x \leq 2 X}\left|[x, x+h k(x)) \cap \mathcal{H}_{1}\right|+O(1) \\
& \leq\left(\sum_{x \in \mathcal{U}_{0}}+\sum_{x \in \mathcal{U}_{1}}+\sum_{x \in \mathcal{U}_{2}}\right)\left|[x, x+h k(x)] \cap \mathcal{H}_{1}\right|+O(1) \\
& \leq X \cdot \delta h / 2+\left|\mathcal{U}_{1}\right| \cdot \frac{h}{\delta^{2}}+\sum_{x \in \mathcal{U}_{2}}\left|[x, x+h k(X)] \cap \mathcal{H}_{1}\right|+O(1)
\end{aligned}
$$

Notice that $n \in \mathcal{H}_{1}$ implies that $w_{n}^{\prime} \geq \varepsilon$ and hence $\lambda_{f}(n) \neq 0$. In particular,

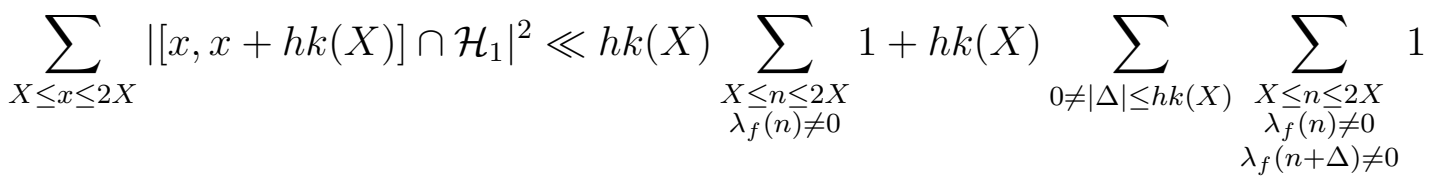

$$
\begin{aligned}
& \ll h^{2} X
\end{aligned}
$$

by a result of Henriot [9, 10], since $\boldsymbol{1}_{\lambda_{f}(n) \neq 0}$ is a multiplicative function of $n$. In addition it follows from this that $\left|\mathcal{U}_{2}\right| \leq C \delta^{4} X$ for some large absolute constant $C>0$. Applying Cauchy-Schwarz and the previous two bounds, we get

$$
\begin{aligned}
\sum_{x \in \mathcal{U}_{2}}\left|[x, x+h k(X)] \cap \mathcal{H}_{1}\right| & \leq\left|\mathcal{U}_{2}\right|^{1 / 2} \cdot\left(\sum_{X \leq x \leq 2 X}\left|[x, x+h k(X)] \cap \mathcal{H}_{1}\right|^{2}\right)^{1 / 2} \\
& \leq C_{0} \delta^{2} h X
\end{aligned}
$$

for some large absolute constant $C_{0}$. Combining the above inequalities it follows that

$$
\delta h X \leq X \delta h / 2+\left|\mathcal{U}_{1}\right| \cdot \frac{h}{\delta^{2}}+C_{0} \delta^{2} X+O(1)
$$

Taking $\delta$ small enough but fixed, we conclude that $\left|\mathcal{U}_{1}\right| \gg X$. The claim now follows since the desired lower bound (5) holds for every $x \in \mathcal{U}_{1}$ by the definitions of $\mathcal{U}_{1}$ and $\mathcal{H}_{1}$

We are now ready to prove Theorem 1.2 which follows from combining Proposition 3.4 and Proposition 3.5. 
Proof of Theorem 1.2. According to Proposition 3.5 we have

$$
\sum_{x \leq n \leq x+h k(x)} w_{n}^{\prime} \geq c h
$$

for a positive proportion $\delta$ of $x \in[X, 2 X]$, with $c>0$ an absolute constant. However, according to Proposition 3.4 we have

$$
\left|\sum_{x \leq n \leq x+h k(x)} \operatorname{sgn}\left(\lambda_{f}(n)\right) w_{n}\right| \leq \frac{C}{\delta} \sqrt{h}
$$

for a proportion of at least $1-\delta^{2}>1-\delta$ of $x \in[X, 2 X]$. Therefore once $h$ is large enough but fixed (larger than $(C /(\delta c))^{2}$ ), we will have a positive proportion of $x \in[X, 2 X]$ for which

$$
\left|\sum_{x \leq n \leq x+h k(x)} \operatorname{sgn}\left(\lambda_{f}(n)\right) w_{n}\right|<\sum_{x \leq n \leq x+h k(x)} w_{n}^{\prime} .
$$

For every such $x$ a sign change of $\lambda_{f}(n)$ must occur in the interval $[x, x+h k(x)]$ since $w_{n} \geq w_{n}^{\prime} \geq 0$ for every $n$. Hence there are $\gg X / k(X)$ sign changes, and the claim follows from Lemma 3.1(ii).

\section{Proof of Proposition 3.2}

We will use the following general bound for mean-values of multiplicative functions.

Lemma 4.1. Let $g: \mathbb{N} \rightarrow[0, \infty)$ be a multiplicative function. Let $A, B$ be constants such that for all $y \geq 1$,

$$
\sum_{p \leq y} g(p) \log p \leq A y \quad \text { and } \quad \sum_{p \in \mathbb{P}} \sum_{\nu \geq 2} \frac{g\left(p^{\nu}\right)}{p^{\nu}} \log p^{\nu} \leq B .
$$

Then, for $x \geq 1$,

$$
\frac{1}{x} \sum_{n \leq x} g(n) \ll(A+B+1) \prod_{p \leq x}\left(1-\frac{1}{p}+\sum_{\nu \geq 1} \frac{g\left(p^{\nu}\right)}{p^{\nu}}\right),
$$

where the implied constant is absolute.

Proof. See [22, Theorem III.3.5].

We will start by proving the upper bound stated in Proposition 3.2. We note that $w_{n}^{\prime 2} \leq g(n)$ where $g(n)$ is a multiplicative function such that

$$
g\left(p^{\nu}\right)= \begin{cases}1 & \text { if } p \leq y, \nu=1 \text { and } \lambda_{f}(p) \neq 0 \\ 0 & \text { if } p \leq y \text { and } \nu>1 \text { or } \lambda_{f}(p)=0 \\ \left|\lambda_{f}\left(p^{\nu}\right)\right|^{2} & \text { if } p>y\end{cases}
$$


By Lemma 4.1 and Lemma 3.1(ii)-(iii),

$$
\sum_{X \leq n \leq 2 X} w_{n}^{\prime 2} \ll X \prod_{\substack{p \leq y \\ \lambda_{f}(p)=0}}\left(1-\frac{1}{p}\right) \prod_{y<p \leq X}\left(1-\frac{1}{p}+\sum_{\nu \geq 1} \frac{\left|\lambda_{f}\left(p^{\nu}\right)\right|^{2}}{p^{\nu}}\right) \ll X \prod_{\substack{p \leq X \\ \lambda_{f}(p)=0}}\left(1-\frac{1}{p}\right) .
$$

We now focus on the lower bound for the first moment of $w_{n}^{\prime}$. The term $w_{n}^{\prime}$ contains at most one summand. Therefore,

$$
\begin{aligned}
\sum_{X \leq n \leq 2 X} w_{n}^{\prime} & =\sum_{\substack{X \leq a b \leq 2 X \\
a \leq y, \lambda_{f}(a) \neq 0 \\
P^{-}(b)>y}} \mu^{2}(a)\left|\lambda_{f}(b)\right| \geq \sum_{2 X / y \leq p \leq 2 X}\left|\lambda_{f}(p)\right| \sum_{\substack{X / p \leq a \leq 2 X / p \\
\lambda_{f}(a) \neq 0}}|\mu(a)|^{2} \\
& \gg X \prod_{\substack{p \leq X \\
\lambda_{f}(p)=0}}\left(1-\frac{1}{p}\right) \sum_{2 X / y \leq p \leq 2 X} \frac{\left|\lambda_{f}(p)\right|}{p} \gg X \prod_{\substack{p \leq X \\
\lambda_{f}(p)=0}}\left(1-\frac{1}{p}\right) \sum_{\substack{2 X / y \leq p \leq 2 X \\
p}} \frac{1}{p}
\end{aligned}
$$

by Lemma 3.1(iv). Hence

$$
\sum_{X \leq n \leq 2 X} w_{n}^{\prime} \gg X \prod_{\substack{p \leq X \\ \lambda_{f}(p)=0}}\left(1-\frac{1}{p}\right) \log \frac{\log 2 X}{\log (2 X / y)} \gg X \prod_{\substack{p \leq X \\ \lambda_{f}(p)=0}}\left(1-\frac{1}{p}\right) .
$$

\section{Proof of Proposition 3.3}

Recall that $\rho^{+}(n)$ are upper bound Brun sieve weights, so that (compare with [3, Section 6.2])

$$
\begin{aligned}
\rho^{+}(n)-\mathbf{1}_{P^{-}(n)>y} & =\sum_{r \geq 0} \sum_{\substack{n=p_{1} \cdots p_{2 r+1} d \\
p_{1}>\cdots>p_{2 r+1}>y_{2 r+1} \\
p_{2 \ell+1} \leq y_{2 \ell+1}(\forall \ell<r) \\
P^{-}(d)>p_{2 r+1}}} \mu(d) \leq \sum_{r \geq 0} \mathbf{1}_{P^{-}(n)>y_{2 r+1}} \mathbf{1}_{\omega(n) \geq 2 r+1} \sum_{k \mid n} \mu(k)^{2} \\
& \leq \sum_{r \geq 0} \mathbf{1}_{P^{-}(n)>y_{2 r+1}} 2^{-(2 r+1)} 2^{2 \omega(n)} .
\end{aligned}
$$

Hence

$$
w_{n}=\sum_{\substack{a b=n \\ a \leq y, \lambda_{f}(a) \neq 0 \\(a, b)=1}} \mu^{2}(a) \rho^{+}(b)\left|\lambda_{f}(b)\right| \leq w_{n}^{\prime}+w_{n}^{\prime \prime}
$$

where $w_{n}^{\prime}$ is as before and

$$
w_{n}^{\prime \prime}:=\sum_{r \geq 0} 2^{-2 r} \sum_{\substack{a b=n \\ a \leq y, \lambda_{f}(a) \neq 0 \\ P^{-}(b)>y_{2 r+1},(a, b)=1}} \mu^{2}(a)\left|\lambda_{f}(b)\right| 2^{2 \omega(b)} .
$$


Note that $w_{n}^{2} \leq 2 w_{n}^{\prime 2}+2 w_{n}^{\prime \prime 2}$. By Proposition 3.2 it is enough to consider $\sum w_{n}^{\prime \prime 2}$. In the sum over divisors of $n$ in the definition of $w_{n}^{\prime \prime}$ we write $a=c d$ with $P^{+}(c) \leq y_{2 r+1}$ and $P^{-}(d)>y_{2 r+1}$. This allows us to re-write the sum over $a b=n$ as follows

$$
\sum_{\substack{c d b=n \\ c d \leq y, \lambda_{f}(c d) \neq 0 \\ P^{-}(b d)>y_{2 r+1} \\ P^{+}(c) \leq y_{2 r+1}}} \mu^{2}(c d)\left|\lambda_{f}(b)\right| 4^{\omega(b)} \leq \sum_{\substack{c \ell=n \\ P^{+}(c) \leq y_{2 r+1} \\ \lambda_{f}(c) \neq 0}} \mu^{2}(c) g_{r}(\ell):=G_{r}(n)
$$

where $g_{r}(\ell)$ is a multiplicative function such that

$$
\begin{aligned}
g_{r}\left(p^{\nu}\right):= & \sum_{\substack{p^{\nu}=d b \\
P^{-}(b d)>y_{2 r+1}}} \mu^{2}(d)\left|\lambda_{f}(b)\right| 4^{\omega(b)} \\
= & \begin{cases}1+4\left|\lambda_{f}(p)\right| & \text { if } p \geq y_{2 r+1} \text { and } \nu=1 \\
4\left|\lambda_{f}\left(p^{\nu}\right)\right|+4\left|\lambda_{f}\left(p^{\nu-1}\right)\right| & \text { if } p \geq y_{2 r+1} \text { and } \nu \geq 2 . \\
0 & \text { otherwise }\end{cases}
\end{aligned}
$$

By Cauchy-Schwarz and (7),

$$
\sum_{X \leq n \leq 2 X} w_{n}^{\prime \prime 2} \ll \sum_{r \geq 0} 2^{-2 r} \sum_{X \leq n \leq 2 X} G_{r}(n)^{2}
$$

Note that $G_{r}(n)^{2}$ is also multiplicative, and

$$
G_{r}(p)^{2}= \begin{cases}\left(1+4\left|\lambda_{f}(p)\right|\right)^{2} \leq 4+64\left|\lambda_{f}(p)\right|^{2} & \text { if } p>y_{2 r+1} \\ 0 & \text { if } p \leq y_{2 r+1} \text { and } \lambda_{f}(p)=0 \\ 1 & \text { if } p \leq y_{2 r+1} \text { and } \lambda_{f}(p)=1\end{cases}
$$

and $G_{r}\left(p^{\nu}\right)^{2} \leq 64 p^{7 \nu / 32}$ by Lemma 3.1(iii). By Lemma 4.1 and Lemma 3.1(iii) we get

$$
\begin{aligned}
\sum_{X \leq n \leq 2 X} G_{r}(n)^{2} & \ll X \prod_{\substack{p \leq y_{2 r+1} \\
\lambda_{f}(p)=0}}\left(1-\frac{1}{p}\right) \cdot \prod_{y_{2 r+1} \leq p \leq X}\left(1+\frac{4+64\left|\lambda_{f}(p)\right|^{2}}{p}\right) \\
& \ll X \prod_{\substack{p \leq X \\
\lambda_{f}(p)=0}}\left(1-\frac{1}{p}\right) \cdot\left(\frac{\log X}{\log y_{2 r+1}}\right)^{69}
\end{aligned}
$$

Combining the above equations and recalling that $y_{r}=X^{\delta / 2\left(1-\gamma^{2}\right) \gamma^{m-1}}$ we obtain

$$
\sum_{X \leq n \leq 2 X} w_{n}^{\prime \prime 2} \ll X \prod_{\substack{p \leq X \\ \lambda_{f}(p)=0}}\left(1-\frac{1}{p}\right) \sum_{r \geq 0} 2^{-2 r}\left(\frac{1}{\left(1-\gamma^{2}\right) \delta \gamma^{2 r}}\right)^{69}
$$


Picking $\gamma=2^{-1 / 100}$ we see that the sum over $r$ is $O(1)$. Combining the previous bounds then leads to

as was claimed.

$$
\sum_{X \leq n \leq 2 X} w_{n}^{2} \ll X \prod_{\substack{p \leq X \\ \lambda_{f}(p)=0}}\left(1-\frac{1}{p}\right)
$$

\section{Proof of Proposition 3.4}

An important role in the proof of Proposition 3.4 is played by estimates for shifted convolution sums.

Lemma 6.1. There exists a small $\delta>0$ such that, uniformly in $a, b, A, B, h \leq x^{\delta}$,

$$
\sum_{\substack{x \leq a A m, b B n \leq 2 x \\ a A m-b B n=h}} \lambda_{f}(A m) \lambda_{f}(B n) \ll x^{1-\delta} .
$$

Proof. Notice first that, for any integer $r$,

$$
\begin{aligned}
f_{r}(z) & :=\sum_{r \mid n} \lambda_{f}(n) n^{(\kappa-1) / 2} e(n z)=\sum_{n} \lambda_{f}(n) n^{(\kappa-1) / 2} \cdot\left(\frac{1}{r} \sum_{0 \leq j<r} e(j n / r)\right) e(n z) \\
& =\frac{1}{r} \sum_{0 \leq j<r} f(z+j / r)
\end{aligned}
$$

is a cusp form of weight $\kappa$ and level $r^{2}$ (see for instance [11, Proof of Proposition 14.19] for a related argument, noting that in the matrix identity below [11, (14.52)] the upper right corner of the right-most matrix should be $d^{2} u / r$ ).

We can re-write the left-hand side of (8) as

$$
\sum_{\substack{x \leq a m, b n \leq 2 x \\ a m-b n=h}} \lambda_{f_{A}}(m) \lambda_{f_{B}}(n)
$$

where $\lambda_{f_{A}}(n)=\lambda_{f}(n)$ if $A \mid n$ and $\lambda_{f_{A}}(n)=0$ otherwise. By a result of Harcos $[8$, Theorem 1] this is indeed bounded by $x^{1-\delta}$. The implicit constant in Harcos's result depends polynomially on the level and weight of the form (see [8, Addendum in end of Section 1]) and furthermore it depends on the implicit constants in Wilton's estimate

$$
\sum_{n \leq x} \lambda_{f_{A}}(n) e(n \alpha) \ll \sqrt{x} \log x
$$

and in the Rankin Selberg bound

$$
\sum_{n \leq x}\left|\lambda_{f_{A}}(n)\right|^{2} \ll x .
$$


Since $\left|\lambda_{f_{A}}(n)\right| \leq\left|\lambda_{f}(n)\right|$ the second sum is trivially less than $\sum_{n \leq x}\left|\lambda_{f}(n)\right|^{2} \ll x$ with no dependence on $A$. On the other hand, the first sum is by Wilton's bound,

$$
\sum_{n \leq x} \lambda_{f_{A}}(n) e(n \alpha)=\frac{1}{A} \sum_{j<A}\left(\sum_{n \leq x} \lambda_{f}(n) e(n(\alpha+j / r))\right) \ll \sqrt{x} \log x
$$

also with no dependence on $A$, since Wilton's bound $\sum_{n \leq x} \lambda_{f}(n) e(n \alpha) \ll \sqrt{x} \log x$ is uniform in $\alpha \in \mathbb{R}$.

This lemma can be used to prove the following mean square estimate in short intervals.

Lemma 6.2. There are absolute positive constants $c$ and $\eta$ such that uniformly in $h \leq X^{\eta}$,

$$
\int_{X}^{2 X}\left|\sum_{x \leq n \leq x+h k(X)} \operatorname{sgn}\left(\lambda_{f}(n)\right) w_{n}\right|^{2} d x \leq c^{2} h X
$$

Proof. We start by expanding the square obtaining sums over $n_{1}$ and $n_{2}$, writing then $n_{2}-n_{1}=r$, and interchanging the integration and summations. This shows that the left hand side equals

$$
\begin{aligned}
& \sum_{X \leq n_{1} \leq 2 X X} \sum_{|r| \leq h k(X)}(h k(X)-|r|) \operatorname{sgn}\left(\lambda_{f}\left(n_{1}\right)\right) w_{n_{1}} \operatorname{sgn}\left(\lambda_{f}\left(n_{1}+r\right)\right) w_{n_{1}+r} \\
& \quad+O\left(h k(X)\left(\sum_{\substack{\left|n_{1}-X\right| \leq h k(X) \\
\left|n_{2}-X\right| \leq h k(X)}}\left|w_{n_{1}} w_{n_{2}}\right|+\sum_{\substack{\left|n_{1}-2 X\right| \leq h k(X) \\
\left|n_{2}-2 X\right| \leq h k(X)}}\left|w_{n_{1}} w_{n_{2}}\right|\right)\right)
\end{aligned}
$$

The error terms contribute at most

$$
(h k(X))^{3} \max _{n \leq 3 X}\left|w_{n}\right|^{2} \ll X^{4 \eta} X^{7 / 32} \ll X^{1 / 2}
$$

by Lemma 3.1(iii), so we can concentrate on the main term which, by definition of $w_{n}$ equals

$$
\begin{aligned}
& \sum_{0<|r| \leq h k(X)}(h k(X)-|r|) \sum_{\substack{X \leq a b \leq 2 X \\
a^{\prime} \bar{b}^{\prime}-a b=r \\
(a, b)=\left(a^{\prime}, b^{\prime}\right)=1 \\
a, a^{\prime} \leq y}} \mu^{2}(a) \mu^{2}\left(a^{\prime}\right) \operatorname{sgn}\left(\lambda_{f}(a)\right) \operatorname{sgn}\left(\lambda_{f}\left(a^{\prime}\right)\right) \rho^{+}(b) \rho^{+}\left(b^{\prime}\right) \lambda_{f}(b) \lambda_{f}\left(b^{\prime}\right) \\
& +h k(X) \sum_{\substack{X \leq n \leq 2 X \\
\lambda_{f}(n) \neq 0}} w_{n}^{2} .
\end{aligned}
$$


By Proposition 3.3 the contribution from the diagonal term $h k(X) \sum w_{n}^{2}$ is bounded by $\ll h X$. We re-write the off-diagonal terms as

$$
\begin{gathered}
\sum_{0<|r| \leq h k(X)}(h k(X)-|r|) \sum_{a, a^{\prime} \leq y} \mu(a)^{2} \mu\left(a^{\prime}\right)^{2} \operatorname{sgn}\left(\lambda_{f}(a)\right) \operatorname{sgn}\left(\lambda_{f}\left(a^{\prime}\right)\right) \\
\cdot \sum_{\substack{X / a \leq b \leq 2 X / a \\
(a, b)=1 \\
\left(a^{\prime}, b^{\prime}\right)=1 \\
a^{\prime} b^{\prime}-a b=r}} \rho^{+}(b) \rho^{+}\left(b^{\prime}\right) \lambda_{f}(b) \lambda_{f}\left(b^{\prime}\right)
\end{gathered}
$$

We focus on the innermost sum, which we re-write in the following way (after introducing the Moebius function to remove the co-primality conditions),

$$
\sum_{\substack{d\left|a \\ d^{\prime}\right| a^{\prime}}} \mu(d) \mu\left(d^{\prime}\right) \sum_{\substack{X / a \leq b \leq 2 X / a \\ d\left|b, a^{\prime}\right| b^{\prime} \\ b^{\prime}-a b=r}} \rho^{+}(b) \rho^{+}\left(b^{\prime}\right) \lambda_{f}(b) \lambda_{f}\left(b^{\prime}\right) .
$$

Furthermore expanding $\rho^{+}(b)$ according to its definition, we get

$$
\sum_{\substack{d\left|a \\
d^{\prime}\right| a^{\prime}}} \mu(d) \mu\left(d^{\prime}\right) \sum_{e, e^{\prime} \in \mathcal{D}^{+}} \mu(e) \mu\left(e^{\prime}\right) \sum_{\begin{array}{r}
X / a \leq b \leq 2 X / a \\
{[d, e] \mid b} \\
{\left[d^{\prime}, e^{\prime}\right] \mid b^{\prime}} \\
a^{\prime} b^{\prime}-a b=r
\end{array}} \lambda_{f}(b) \lambda_{f}\left(b^{\prime}\right) .
$$

Notice that $\mathcal{D}^{+} \subset[1, y]$, and that $d, d^{\prime} \leq y$ since $a, a^{\prime} \leq y$. Therefore the inner sum is by Lemma 6.1 bounded by $\ll X^{1-\eta}$ for some absolute $\eta>0$, provided that $y=X^{\delta}$ is chosen small enough. Combining the above equations it follows that the contribution of the off-diagonal terms is bounded by $\ll(h k(X))^{2} y^{4+\varepsilon} X^{1-\eta}$ and this is $\ll X^{1-\kappa}$ for some $\kappa>0$ provided that $\delta>0$ is chosen small enough.

Proof of Proposition 3.4. By previous lemma and Chebychev's inequality the measure of the set of $x \in[X, 2 X]$ for which

$$
\left|\sum_{x \leq n \leq x+h k(x)} \operatorname{sgn}\left(\lambda_{f}(n)\right) w_{n}\right| \geq c K \sqrt{h}
$$

is at most $X / K^{2}$.

\section{Proof of Corollary 1.3}

Write $g(n)=\operatorname{sgn}\left(\lambda_{f}(n)\right)$, so that $g(n)$ is a multiplicative function taking values in $\{-1,0,1\}$. The upper bound

$$
\sum_{n \leq x} g(n) g(n+1) \leq(1-c) x .
$$


follows immediately from Theorem [1.2, To obtain the lower bound

$$
\sum_{n \leq x} g(n) g(n+1) \geq(-1+c) x
$$

we first note that, by [5, Lemma 4.1], there is an even integer $b \ll_{f} 1$ such that $g\left(2^{b}\right)=1$. Notice that the claim is trivial unless $g\left(2^{j}\right) \neq 0$ for all $j \leq b$. If $g(2)=-1$, the existence of such $b$ implies that there is an integer $j<b$ such that $g\left(2^{j}\right)=-1$ and $g\left(2^{j+1}\right)=1$. On the other hand if $g(2)=1$, then, since $b$ is even, there must be an integer $j<b$ such that $g\left(2^{j}\right)=g\left(2^{j+1}\right)$. Hence in any case we find $j \ll 1$ such that $g\left(2^{j+1}\right)=g(2) g\left(2^{j}\right)$.

Consider now $n \equiv 2^{j}-1\left(\bmod 2^{j+1}\right)$. Notice that, for such $n, g(2 n)=g(2) g(n)$ and

$$
g(2 n+2)=g\left(2^{j+1}\right) g\left((n+1) / 2^{j}\right)=g(n+1) g\left(2^{j+1}\right) / g\left(2^{j}\right)=g(2) g(n+1),
$$

so that

$g(n) g(n+1) g(2 n) g(2 n+1) g(2 n+1) g(2 n+2)=(g(n) g(n+1) g(2) g(2 n+1))^{2} \geq 0$.

Hence one of $g(n) g(n+1), g(2 n) g(2 n+1)$ and $g(2 n+1) g(2 n+2)$ must be nonnegative, and (9) follows since positive proportion of $n \leq x / 2$ satisfy $n \equiv 2^{j}-1$ $\left(\bmod 2^{j+1}\right)$.

\section{ACKNOWLEDGMENTS}

The authors would like to thank the anonymous referee for suggesting a complete re-write of the proof (a variant of which is presented here). The referee's argument led to several improvements and to a much cleaner proof, for which the authors are extremely grateful. The authors would also like to thank Andrew Granville for providing the proof of Lemma 2.1 and Matti Jutila for mentioning the shifted convolution problem. They are also grateful to Jie Wu and Wenguang Zhai for an e-mail discussion concerning [23] and to Eeva Vehkalahti for helpful comments on earlier versions of this manuscript. They also would like to thank Peter Sarnak for a Lemma which was used in the previous version of the paper and for posing the question on the entropy of $\lambda_{f}(n)$. Finally the authors are grateful to Brian Conrey for suggesting the use of a mollifier at the October 2013 meeting in Oberwolfach.

\section{REFERENCES}

[1] Y. J. Choie, A. Sankaranarayanan, and J. Sengupta. On the sign changes of Hecke eigenvalues. In Number theory and applications, pages 25-32. Hindustan Book Agency, New Delhi, 2009.

[2] P. D. T. A. Elliott and J. Kish. Harmonic Analysis on the Positive Rationals II: Multiplicative Functions and Maass Forms. arXiv:1405.7132.

[3] J. Friedlander and H. Iwaniec. Opera de cribro, volume 57 of American Mathematical Society Colloquium Publications. American Mathematical Society, Providence, RI, 2010. 
[4] S. Gelbart and H. Jacquet. A relation between automorphic representations of GL(2) and GL(3). Ann. Sci. École Norm. Sup. (4), 11(4):471-542, 1978.

[5] A. Ghosh and P. Sarnak. Real zeros of holomorphic Hecke cusp forms. J. Eur. Math. Soc. (JEMS), 14(2):465-487, 2012.

[6] A. Granville, D. Koukoulopoulos, and K. Matomäki. When the sieve works. Duke Math. J., To appear, pre-print available at http://users.utu.fi/ksmato/papers/SieveWorks.pdf.

[7] R. R. Hall and G. Tenenbaum. Effective mean value estimates for complex multiplicative functions. Math. Proc. Cambridge Philos. Soc., 110(2):337-351, 1991.

[8] G. Harcos. An additive problem in the Fourier coefficients of cusp forms. Math. Ann., $326(2): 347-365,2003$.

[9] Kevin Henriot. Nair-Tenenbaum bounds uniform with respect to the discriminant. Math. Proc. Cambridge Philos. Soc., 152(3):405-424, 2012.

[10] Kevin Henriot. Nair-Tenenbaum uniform with respect to the discriminant-Erratum. Math. Proc. Cambridge Philos. Soc., 157(2):375-377, 2014.

[11] H. Iwaniec and E. Kowalski. Analytic number theory, volume 53 of American Mathematical Society Colloquium Publications. American Mathematical Society, Providence, Rhode Island, 2004.

[12] H. H. Kim. Functoriality for the exterior square of $\mathrm{GL}_{4}$ and the symmetric fourth of $\mathrm{GL}_{2}$. J. Amer. Math. Soc., 16(1):139-183, 2003. With appendix 1 by Dinakar Ramakrishnan and appendix 2 by Kim and Peter Sarnak.

[13] H. H. Kim and F. Shahidi. Cuspidality of symmetric powers with applications. Duke Math. J., 112(1):177-197, 2002.

[14] E. Kowalski, Y.-K. Lau, K. Soundararajan, and J. Wu. On modular signs. Math. Proc. Cambridge Philos. Soc., 149(3):389-411, 2010.

[15] Y.-K. Lau, J.Y. Liu, and J. Wu. Coefficients of symmetric square L-functions. Sci. China Math., 53(9):2317-2328, 2010.

[16] Y.-K. Lau and J. Wu. The number of Hecke eigenvalues of same signs. Math. Z., 263(4):959970, 2009.

[17] K. Matomäki and M. Radziwiłł. Multiplicative functions in short intervals. Preprint, available at http://arxiv.org as arXiv:1501.04585 [math.NT].

[18] J. Meher, K. D. Shankhadhar, and G. K. Viswanadham. A short note on sign changes. Proc. Indian Acad. Sci. Math. Sci., 123(3):315-320, 2013.

[19] M. R. Murty. Oscillations of Fourier coefficients of modular forms. Math. Ann., 262(4):431-446, 1983.

[20] P. Sarnak and A. Ubis. The horocycle flow at prime times. arxiv: 1110.077\%.

[21] J.-P. Serre. Quelques applications du théorème de densité de Chebotarev. Inst. Hautes Études Sci. Publ. Math., 54:323-401, 1981.

[22] G. Tenenbaum. Introduction to Analytic and Probabilistic Number Theory. Cambridge University Press, 1995.

[23] J. Wu and W. Zhai. Distribution of Hecke eigenvalues of newforms in short intervals. Q. J. Math., 64(2):619-644, 2013. 
Department of Mathematics and Statistics, University of Turku, 20014 Turku, FINLAND

E-mail address: ksmato@utu.fi

School of Mathematics, Institute for Advanced Study, 1 Einstein Drive, PrinceTON, NJ, 08540, USA

E-mail address: maksym@ias.edu

Current address: Department of Mathematics, Rutgers University, Hill Center - Busch Campus, 110 Frelinghuysen Road, Piscataway, NJ 08854-8019, USA

E-mail address: maksym.radziwill@gmail.com 